\title{
Immune-Enhancing Effects of Lactobacillus plantarum 200655 Isolated from Korean Kimchi in a Cyclophosphamide-Induced Immunocompromised Mouse Model
}

\author{
Kyeong Jin Kim', Hyun-Dong Paik ${ }^{2}$, and Ji Yeon Kim ${ }^{1,3 *}$ \\ ${ }^{1}$ Department of Nano Bio engineering, Seoul National University of Science and Technology, Seoul 01811, Republic \\ of Korea \\ ${ }^{2}$ Department of Food Science and Biotechnology of Animal Resources, Konkuk University, Seoul 05029, Republic of Korea \\ ${ }^{3}$ Department of Food Science and Technology, Seoul National University of Science and Technology, Seoul 01811, \\ Republic of Korea
}

\begin{abstract}
In this study, we evaluated the immune-enhancing activity of kimchi-derived Lactobacillus plantarum 200655 on immune suppression by cyclophosphamide (CP) in ICR mice. Animals were fed distilled water or $1 \times 10^{9}$ colony-forming unit/kg B.W. 200655 or Lactobacillus rhamnosus GG as a positive control for 14 days. An in vivo model of immunosuppression was induced using CP 150 and $100 \mathrm{mg} / \mathrm{kg} \mathrm{B.W.}$ at 7 and 10 days, respectively. Body weight, spleen index, spleen weight, and gene expression were measured to estimate the immune-enhancing effects. The dead 200655 (D-200655) group showed an increased spleen weight compared to the sham control (SC) group. Similarly, the spleen index was significantly higher than that in the CP-treated group. The live 200655 (L-200655) group showed an increased mRNA expression of tumor necrosis factor-alpha (TNF- $a$ ) and interleukin (IL)- 6 in splenocytes. Also, the iNOS and COX-2 mRNA expression was upregulated in the L-200655 group compared to the CP-only (SC) group. The phosphorylation of ERK and MAPK was also upmodulated in the L-200655 group. These results indicate that $L$. plantarum 200655 ameliorated CP-induced immune suppression, suggesting that $L$. plantarum 200655 may have the potential to enhance the immune system.
\end{abstract}

Keywords: Cyclophosphamide, splenocytes, Lactobacillus plantarum, immune enhancement, MAPK/ERK signaling pathway

Received: March 17, 2021 Accepted: April 2, 2021

First published online: April 5, 2021

*Corresponding author Phone: +82-2-970-6740 E-mail: jiyeonk@seoultech.ac.kr

pISSN 1017-7825 eISSN 1738-8872

Copyright(C) 2021 by The Korean Society for Microbiology and Biotechnology

\section{Introduction}

The immune system plays a crucial defense-mechanism role by preventing pathogens and various infectious agents from initiating disease [1]. Homeostasis in the immune system is maintained by immunostimulation and immunosuppression, leading to immune regulation [2]. This defense system classically comprises the two sectors of innate and adaptive immunity [3]. In the non-specific defenses, known as innate immunity, neutrophils, macrophages, natural killer (NK) cells, and dendritic cells perceive exogenous factors and promote the production of cytokines [4]. When macrophages are activated, they eliminate the hazard directly by phagocytosis and indirectly by releasing inflammatory cytokines such as nitric oxide $(\mathrm{NO})$, prostaglandin $\mathrm{E}_{2}\left(\mathrm{PGE}_{2}\right)$, tumor necrosis factor- $\alpha$ (TNF- $\alpha$ ), and the interleukins IL-1 $\beta$ and IL-6 [5]. In contrast, the specific defenses, known as adaptive immunity, are characterized by $\mathrm{T}$ and $\mathrm{B}$ cells [6]. Inflammatory cytokines are also accompanied by the NF- $\kappa B$ and MAPK signaling pathways [7], which are mediated by toll-like receptors (TLRs) [8]. Physiological and pathological responses, including stress responses, inflammation, and apoptosis, are mediated by the MAPK signaling pathway in mammalian cells and tissues [9]. In the MAPK signaling pathway, phosphorylation of p38 MAPK and ERK1/2 plays important roles, activating the production of TNF- $\alpha$, IL-6, and IL-8, which are inflammatory factors [10]. Moreover, the MAPK cascade and IL-8 secretion are triggered by TNF- $\alpha$ [11]. In addition to MAPK, NF- $\mathrm{KB}$ regulates the expression of proinflammatory mediators. NF- $\mathrm{kB}$ p 65 protein combines with I $\kappa B$ protein and exists in the cytoplasm. Depending on external stimuli, NF- $\kappa B$ kinases (IKKs) can phosphorylate the IкB family of proteins, followed by ubiquitination and degradation, further phosphorylating p65 and releasing it into the nucleus, where it promotes inflammation.

Cyclophosphamide (CP) is a widely used alkylating cytotoxic agent that exerts broad-spectrum activities in modulating immune responses against various illnesses $[1,12]$. However, administration of high-dose $\mathrm{CP}$ can cause immunosuppression and intestinal complications [13]. CP-induced immunosuppression decreases the number 
of lymphocytes in the blood and bone marrow, which results in inhibition of cytokine release from T cells [14].

Recently, it has become popular for immunocompromised people to ingest foods containing probiotics to enhance their immune systems [15]. Lactic acid bacteria (LAB) are gram-positive, non-spore forming, nonrespiring cocci or rods that ferment yogurt, kimchi, and cheese [16]. Lactobacillus species, which can regulate the immune system and strengthen host defense against pathogens, are the main LAB in Korean kimchi, a traditional Korean fermented vegetable [17]. Among lactobacilli, Lactobacillus plantarum is a probiotic with immunomodulatory effects that can mitigate chronic intestinal inflammation and irritable bowel syndrome [18]. Lactobacillus species also promote the expression of IL-12 and interferon (IFN)- $\gamma$ in monocytes and induce their differentiation into type 1 helper (TH1) T cell subsets [19]. In addition, previous studies reported that live L. plantarum HY7712 isolated from kimchi reverses the inhibition of NK and T cell activities caused by CP and c-irradiation in mice [16, 20].

The development of LAB with immune-improving properties is helpful for immunosuppressed people because of the lack of side effects. Recently, the inactivated form, as well as the live type, has been found to be beneficial for human health [21]. Although the mechanism is still unclear, many studies have reported that heat-killed LAB could show immunomodulatory activities. Likewise, studies have indicated the immune-enhancing effect of L. plantarum derived from kimchi on immunosuppressed mice, but the underlying mechanism remains poorly understood. The beneficial effects of probiotics depend on their species- and strain-specific characteristics [22]. Therefore, the immune-enhancing activity of $L$. plantarum 200655 must be investigated. The aim of this study was to elucidate the immuno-enhancing potential of live and dead LAB in immunosuppression of CP-treated mice with a focus on the MAPK signaling pathway.

\section{Materials and Methods \\ Materials}

Roswell Park Memorial Institute (RPMI) 1640 medium was purchased from Gibco-Life Technologies (USA); fetal bovine serum (FBS) and penicillin-streptomycin mixture from Biowest (France). Cyclophosphamide, lipopolysaccharide (LPS), and trypan blue were obtained from Sigma-Aldrich (USA).

\section{Preparation and Identification of LAB Strains}

L. plantarum 200655 was provided by Konkuk University (Korea) and prepared as follows [23]: L. plantarum 200655 was isolated from cabbage kimchi. The kimchi sample ( $1 \mathrm{~g})$ was serially diluted and inoculated on de Man, Rogosa, and Sharpe (MRS) agar at $37^{\circ} \mathrm{C}$ for $24 \mathrm{~h}$. A colony was inoculated and incubated in MRS broth under the same condition. The commercial probiotic strain L. rhamnosus GG was obtained from the Korean Collection for Type Cultures (KCTC, Korea) and prepared as follows: LAB strains were incubated in MRS broth at $37^{\circ} \mathrm{C}$ for $18 \mathrm{~h}$ before use. To harvest the $\mathrm{LAB}$ cells, bacterial cell cultures were centrifuged at $14,240 \times g$ at $4^{\circ} \mathrm{C}$ for $10 \mathrm{~min}$. The bacterial cells were washed thrice and resuspended in phosphate-buffered saline (Gibco, USA). The dead LAB was prepared by heating the harvested cells at $100^{\circ} \mathrm{C}$ for $1 \mathrm{~h}$. L. plantarum 200655 was identified by $16 \mathrm{~S}$ rRNA gene conducted by Bionics Inc. (Korea). The sequencing results were analyzed by comparison with the GenBank database using the Basic Local Alignment Search Tool (BLAST) website (http://blast.ncbi.nlm.nih.gov).

\section{Cyclophosphamide-Induced Immunosuppression Mouse Model}

Seven-week-old ICR mice were purchased from Orient Bio (Korea) and maintained under a regular 12-h light/ 12 -h dark cycle at $21-24^{\circ} \mathrm{C}$, with relative humidity of $45 \%-60 \%$, and a commercial diet. After 1 week of acclimation, the mice were randomly divided into five groups $(n=10)$ as follows: normal control (NC), CP only (SC), CP $+1 \times$ $10^{9} \mathrm{CFU} / \mathrm{kg}$ B.W. L. rhamnosus GG as positive control (PC), CP $+1 \times 10^{9} \mathrm{CFU} / \mathrm{kg}$ B.W. live L. plantarum 200655 (L-200655), and CP $+1 \times 10^{9} \mathrm{CFU} / \mathrm{kg}$ B.W. dead L. plantarum 200655 (D-200655). The NC and SC groups were administered distilled water via oral gavage. Forty mice were injected intraperitoneally (i.p.) with CP in sterile saline $(150$ and $100 \mathrm{mg} / \mathrm{kg}$ ) for 7 and 10 days to establish immunosuppression models. The experimental procedures (P204004) were approved by the Institutional Animal Care and Use Committee (IACUC) of KPC laboratory (Non-clinical contract research organization, Korea).

\section{NK Cell Activity Analysis}

On day 14 , whole blood from all groups was collected and gently mixed with NK cell-activating reagent, and the mixtures were then incubated for $24 \mathrm{~h}$ at $37^{\circ} \mathrm{C}$. After incubation, each sample was centrifuged for $15 \mathrm{~min}$. The supernatants were then carefully transferred to new tubes. Each supernatant of NK cell activity was measured by determining the levels of IFN- $\gamma$ production using the Murine NK Activity Kit (ATGen, Korea) according to the manufacturer's instructions.

\footnotetext{
Alanine Aminotransferase (ALT) Activity in Serum

Serum ALT activity was determined to assess hepatocellular injury. ALT activity was measured using a colorimetric assay kit (Sigma Chemical) according to the manufacturer's instructions. Briefly, the samples were centrifuged and stored in a freezer at $-80^{\circ} \mathrm{C}$ until analysis. The enzyme activities of ALT in the sera were estimated using a microplate reader (BioTek, USA).

\section{Isolation of Splenocytes and Cell Culture}

The spleens were removed to obtain splenocytes from mice. Next, the spleens were weighed and the spleen index was calculated by the following equation: spleen index $(\%)=[($ spleen weight/body weight $)] \times 100$. The spleens were then gently pressed with a syringe and forced through a $70-\mu \mathrm{m}$ cell strainer (SPL Life Sciences,
} 
Korea). Cells were treated with red blood cell lysis buffer (Sigma-Aldrich), and isolated splenocytes were incubated in RPMI-1640 containing 10\% (FBS) and 1\% antibiotics (penicillin and streptomycin) with LPS in a 5\% $\mathrm{CO}_{2}$ incubator.

\section{RNA Extraction and Quantitative RT-PCR}

Total RNA was isolated from splenocytes using TRIzol reagent (Life Technologies, USA) following the manufacturer's protocol. Total RNA was then reverse-transcribed using a high-capacity cDNA reverse transcription kit. The Universal Probe Library (UPL) method was used to quantify the expression of TNF- $\alpha$, IL-6, IL-1 $\beta$, and GAPDH in splenocytes using a Light Cycler 96 System (Hoffmann La Roche, Switzerland). The relative mRNA expression, normalized to GAPDH levels, was calculated using the comparative CT method. The sequences of the sense and antisense primers used for RT-qPCR analysis were as follows: TNF- $\alpha$ (forward, $5^{\prime}$ tcttctcattcctgcttgtgg-3'; reverse, 5' -ggtctgggccatagaactga-3'); IL-6 (forward, 5' -gctaccaaactggatataatcagga-3'; reverse, $5^{\prime}$-ccaggtagctatggtactccagaa-3'); IL-1 $\beta$ (forward, $5^{\prime}$-agttgacggaccccaaaag- $3^{\prime}$; reverse, $5^{\prime}$ - agctggatgctctcatcagg$3^{\prime}$ ); and GAPDH (forward, 5' -aagagggatgctgcccttac- $3^{\prime}$; reverse, 5' -ccattttgtctacgggacga- $3^{\prime}$ ).

\section{Protein Extraction and Western Blot Analysis}

The cultured splenocytes were lysed by incubation for 20 min on ice in PRO-PREP Protein Extraction Solution (iNtRON Biotechnology, Korea). Protein was added to $2 \times$ sample buffer (Bio-Rad, USA), incubated at $90^{\circ} \mathrm{C}$ for $2 \mathrm{~min}$, and electrophoresed on $10 \%$ sodium dodecyl sulfate (SDS) polyacrylamide gels. The resolved proteins in electrophoretic SDS polyacrylamide gel were transferred to a polyvinylidene difluoride (PVDF) membrane (BioRad). The membrane was blocked with $5 \%$ skim milk for $1 \mathrm{~h}$. The primary antibodies against ERK, phosphorylated ERK (p-ERK), p38 MAPK, and phosphorylated p38 MAPK (Cell Signaling Technology, USA) were added, and the membrane was incubated overnight at $4^{\circ} \mathrm{C}$. After washing the membrane three times with TBST buffer (Tris-buffered saline, $0.1 \%$ Tween 20) for $5 \mathrm{~min}$, the secondary antibody (Cell Signaling) was added, allowed to attach at room temperature for $2 \mathrm{~h}$, and washed three times for $5 \mathrm{~min}$ with TBST buffer. The immunoreactive protein bands were developed with the ClarityTM Western ECL substrate (Bio-Rad) and quantified using a ChemiDoc MP Imaging System, which was followed by analysis using Image Lab software (Bio$\mathrm{Rad})$.

\section{Statistical Analysis}

Data are expressed as the mean \pm standard error of the mean (SEM). Statistical analyses were performed using Duncan's multiple range test, which is a one-way analysis of variance (ANOVA) (SAS 9.4, SAS Institute, USA). Statistical significance was set at $p<0.05$.

\section{Results}

\section{Effect of 200655 on the CP-Induced Mouse Model}

To investigate the immune-enhancing effect of 200655 under physiological conditions, an immunosuppressed mouse model was induced by intraperitoneal administration of CP. The effects of each LAB strain on body and spleen weight and spleen index are shown in Table 1 and Fig. 1, respectively. There was no significant difference in the body weight of all groups on day 0 , on which oral administration of each LAB strain was commenced. On day 7 , body weight increased in all groups. Because $\mathrm{CP}$ was injected, body weight decreased in all groups except for the NC group. In the SC group, body weight was significantly reduced by approximately $4.9 \%$ compared to the NC group on day 14 . The body weights of the PC, L-200655, and D-200655 groups decreased by approximately 5.1\%, $2.2 \%$, and $7.5 \%$, respectively, compared to the SC group. The highest loss in body weight was observed in the D200655 group. Similarly, the NC group showed the highest spleen weight and index. In contrast, the reduction in spleen weight caused by CP significantly improved in mice administered D-200655. Likewise, the spleen index was significantly increased in the D-200655 group compared with that in the SC group.

\section{Whole Blood NK Activity and Serum ALT Levels in the CP-Induced Mouse Model}

As shown in Table 2, IFN- $\gamma$ levels from NK cells were decreased in SC group $(163.10 \pm 20.55 \mathrm{pg} / \mathrm{ml})$ compared to NC group $(212.97 \pm 36.98 \mathrm{pg} / \mathrm{ml})$. However, the suppression of NK cell activity by CP was recovered by treatment with 200655 (L-200655, $170.09 \pm 19.76$ pg/ml; D-200655, $175.64 \pm 12.44 \mathrm{pg} / \mathrm{ml}$ ), but there was no significant

Table 1. Effect of 200655 on body weight in CP-induced immunosuppressed mice.

\begin{tabular}{cccc}
\hline \multirow{2}{*}{ Group } & \multicolumn{3}{c}{ Body weight (g) } \\
\cline { 2 - 4 } & Day 0 & Day 7 & Day 14 \\
\hline NC & $36.10 \pm 0.31$ & $37.33 \pm 0.54$ & $34.73 \pm 0.55^{\mathrm{a}}$ \\
SC & $36.08 \pm 0.29$ & $37.34 \pm 0.43$ & $33.41^{\mathrm{ab}}$ \\
PC & $36.07 \pm 0.27$ & $36.20 \pm 0.39^{\mathrm{ab}}$ \\
L-200655 & $36.07 \pm 0.27$ & $36.87 \pm 0.38$ & $33.70 \pm 0.44^{\mathrm{ab}}$ \\
D-200655 & $36.03 \pm 0.25$ & $36.64 \pm 0.39$ & $32.93 \pm 0.64^{\mathrm{b}}$ \\
\hline
\end{tabular}

NC, normal control; SC, sham control; PC, positive control; L-200655, live 200655 lactic acid bacteria; D-200655, dead 200655 lactic acid bacteria. Values with different letters within a row are significantly different at $p<0.05$, as determined by Duncan's multiple range test. Values from small to large are arranged in alphabetical order. 

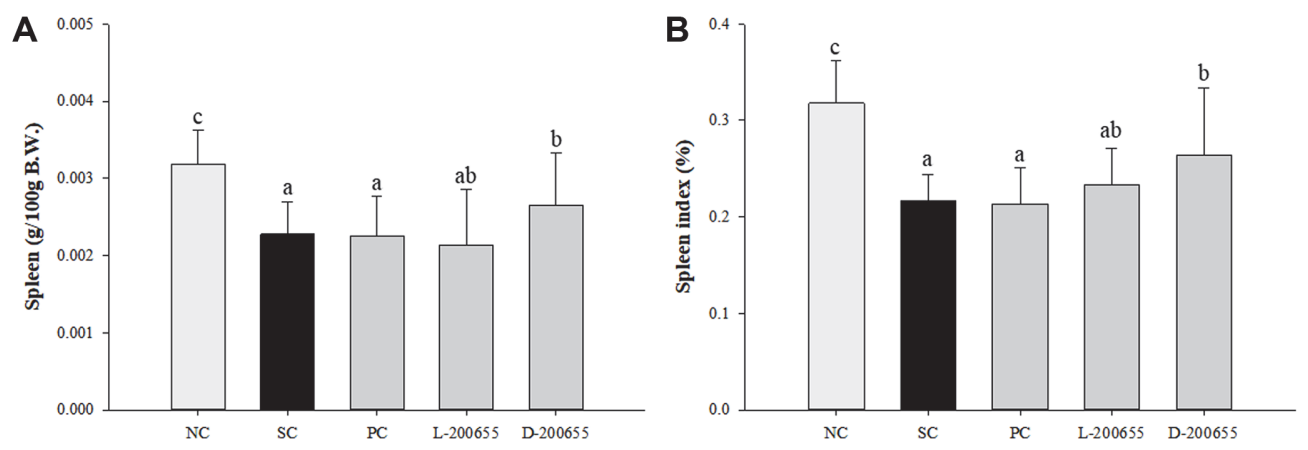

Fig. 1. Regulatory effect of 12204 on spleen weight (A) and index (B) of CP-induced immunosuppressed mice. NC, normal control; SC, sham control; PC, positive control; L-200655, live 200655 lactic acid bacteria; D-200655, dead 200655 lactic acid bacteria. Values with different letters within a row are significantly different at $p<0.05$, as determined by Duncan's multiple range test. Values from small to large are arranged in alphabetical order.

Table 2. Effect of 200655 on NK cell and alanine aminotransferase activity in CP-induced immunosuppressed mice.

\begin{tabular}{ccc}
\hline Group & IFN- $\gamma(\mathrm{pg} / \mathrm{ml})$ & ALT $(\mathrm{mU} / \mathrm{ml})$ \\
\hline NC & $212.97 \pm 36.98$ & $38.97 \pm 18.97$ \\
SC & $163.10 \pm 20.55$ & $40.75 \pm 19.79$ \\
PC & $154.11 \pm 9.43$ & $33.94 \pm 10.66$ \\
L-200655 & $170.09 \pm 19.76$ & $39.29 \pm 12.12$ \\
D-200655 & $175.64 \pm 12.44$ & $36.70 \pm 15.09$ \\
\hline
\end{tabular}

NC, normal control; SC, sham control; PC, positive control; L-200655, live 200655 lactic acid bacteria; D-200655, dead 200655 lactic acid bacteria. Values with different letters within a row are significantly different at $p<0.05$, as determined by Duncan's multiple range test. Values from small to large are arranged in alphabetical order.

difference. The effects of 200655 on CP-induced elevation of serum ALT activity are shown in Table 2. Increased ALT activity by CP in all sample groups was alleviated compared with the SC group, but the difference was not significant.

\section{Effect of 200655 on Inflammatory Cytokine Expression in Splenocytes}

To test the immune-improving effect of 200655 on splenocytes, RT-qPCR was conducted for all experimental groups. The outcomes reconfigured the case in which SC significantly reduced the levels of inflammatory mRNA expression, namely, TNF- $\alpha$, IL-6, and IL-1 $\beta$ (Fig. 2). In splenocytes from the SC group, the expression of proinflammatory cytokines sharply decreased by approximately $40 \%, 48 \%$, and $88 \%$ in comparison to that in the NC group. In contrast, the L-200655 and D-200655 groups showed higher relative mRNA expression of TNF- $\alpha$ by about 1.7- and 1.4-fold, respectively, compared to the SC group. Similarly, relative mRNA expression of IL-6 was recovered by approximately 1.4- and 1.4-fold in the L-200655 and D-200655 groups, respectively, compared to the SC group. Although L-200655 showed an increased tendency of IL-1 $\beta$ mRNA expression, there was no significant difference in all groups except for the NC group. The mRNA expression of these cytokines was almost recovered in L-200655 compared to D-200655.

A

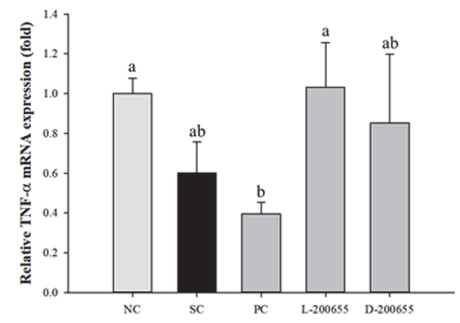

B

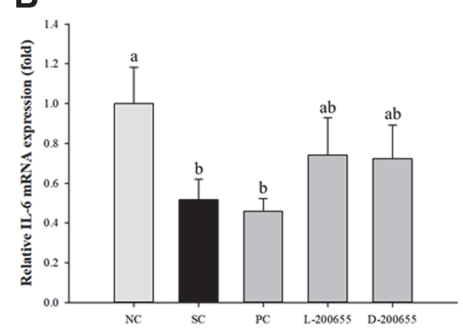

C

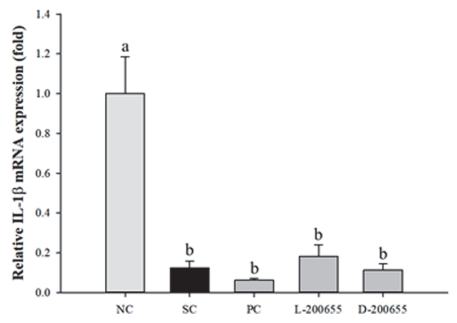

Fig. 2. Relative mRNA expression profile of proinflammatory cytokines in splenocytes treated with LPS as mitogen in CP-induced immunosuppressed mice. (A) TNF- $\alpha$; (B) IL-6; (C) IL-1 $\beta$. NC, normal control; SC, sham control; PC, positive control; L-200655, live 200655 lactic acid bacteria; D-200655, dead 200655 lactic acid bacteria. Values with different letters within a row are significantly different at $p<0.05$, as determined by Duncan's multiple range test. Values from small to large are arranged in alphabetical order. 
A

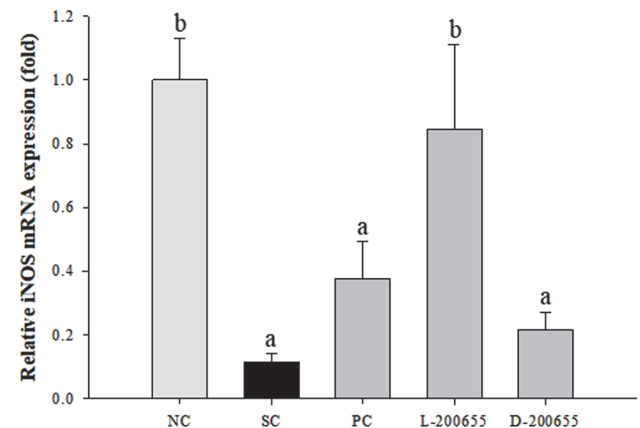

B

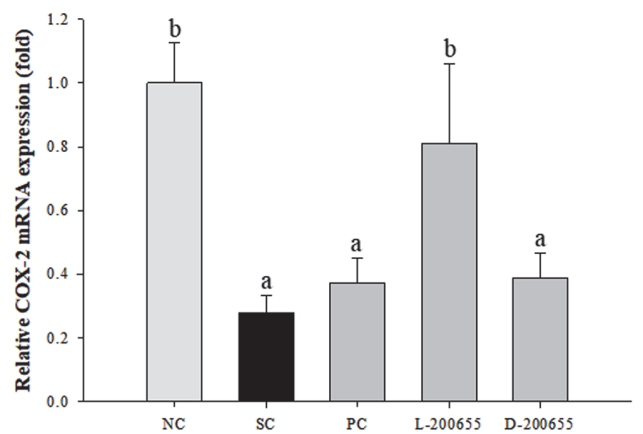

Fig. 3. Relative mRNA expression of proinflammatory mediators in splenocytes treated with LPS as mitogen in CP-induced immunosuppressed mice. (A) iNOS; (B) COX-2; NC, normal control; SC, sham control; PC, positive control; L-200655, live 200655 lactic acid bacteria; D-200655, dead 200655 lactic acid bacteria. Values with different letters within a row are significantly different at $p<0.05$, as determined by Duncan's multiple range test. Values from small to large are arranged in alphabetical order.
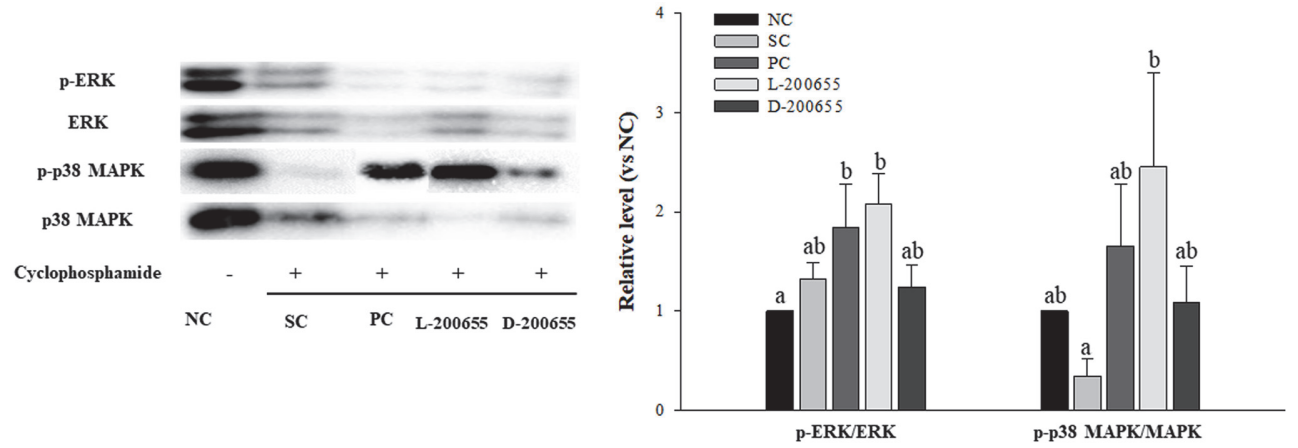

Fig. 4. Effect of 200655 on MAPK signaling pathway in splenocytes treated with LPS as mitogen in CP-induced immunosuppressed mice. NC, normal control; SC, sham control; PC, positive control; L-200655, live 200655 lactic acid bacteria; D-200655, dead 200655 lactic acid bacteria. Values with different letters within a row are significantly different at $p<$ 0.05 , as determined by Duncan's multiple range test. Values from small to large are arranged in alphabetical order.

\section{Activation of the MAPK Signaling Pathway}

In addition to the inflammatory cytokine levels, iNOS and COX-2 mRNA levels were confirmed as immuneenhancing responses. In the SC group, the iNOS and COX-2 mRNA levels decreased by approximately $89 \%$ and $72 \%$, respectively, compared to the NC group (Fig. 3). In contrast, each of the three LAB groups displayed increased relative mRNA expression of iNOS by approximately 3.3-, 7.4-, and 1.9-fold in the PC, L-200655, and D200655 groups, respectively, compared to the SC group. However, there was no significant difference between the PC and D-200655 groups. Likewise, the relative mRNA expression of COX-2 was increased by approximately 1.3-, 2.9-, and 1.4-fold in the PC, L-200655, and D-200655 groups, respectively, compared to the SC group. However, there was no significant difference between the PC and D-200655 groups. The phosphorylation of ERK was upregulated by approximately 1.3 -fold in the SC group compared to the NC group (Fig. 4). After administration of LAB, the phosphorylation of ERK was also observed, except for the D-200655 group, by approximately 1.3- and 1.6-fold in the PC and L-200655 groups, respectively, compared to the SC group. In terms of p38 MAPK, the SC group showed decreased phosphorylation of p38 MAPK by approximately $65 \%$ compared to the NC group. Meanwhile, each of the three LAB groups was upregulated by approximately 4.7-, 7-, and 3.1-fold in the PC, L200655 , and D-200655 groups, respectively, compared to the SC group.

\section{Discussion}

Various studies have shown that bioactive compounds promote immune responses when the body's immune function is degraded owing to infection or disease. In accordance with emerging issues of biological stability for chemotherapy and immuno-disease treatment, which have side effects, harmless substances such as plants, fruits, and LAB have been studied as immune regulators as well as for their health-promoting properties. Therefore, this study was performed to verify and support the hypothesis that LAB derived from kimchi could modulate activation of the MAPK signaling pathway in a mouse model featuring deteriorated immunity.

$\mathrm{CP}$ is a well-known immunosuppressive drug used in clinical research that damages DNA structure, kills immune cells, interferes with proliferation and differentiation of macrophages of $\mathrm{T}$ and $\mathrm{B}$ cells, and restrains the humoral and cellular immune responses [24]. In the long term, high-dose CP treatment triggers immunosuppression accompanied by a reduction in body weight, relative weight of the spleen and thymus, immune system factors, lymphocyte proliferation, and NK cell activity [25]. In this study, the changes in body 
weight and spleen were confirmed by CP. Compared with the NC group, the SC group that received CP showed a significant decrease in body weight. Similarly, each LAB-treated group showed a noticeable reduction in weight. These results suggest that the injection of $\mathrm{CP}$ had a greater influence on apoptosis in spleen cells than LAB had on cell proliferation [26]. As a negative influence, spleen weight was significantly lower in the SC group than in the NC group. In the same vein, the spleen index also decreased in the SC group. However, both the L-200655 and D200655 groups significantly inhibited the reduction of spleen weight and index. They can therefore have a positive effect on the immune system. CP challenge also induces liver damage, which was revealed by increased levels of ALT and AST in the serum $[27,28]$. The SC group showed an increase in ALT concentration compared to that in the NC group. These results suggest that CP treatment has considerable hepatotoxic effects. In this study, although the PC and D-200655 groups showed low levels of ALT, there were no significant differences.

NK cells are involved in the first step of the reaction to infection and play an important role in innate immune responses $[29,30]$. Major proinflammatory cytokines such as IFN- $\gamma$ and TNF- $\alpha$ are released from NK cells and modulate immune responses in infected cells [31]. Previous study reported that patients with diseases have considerably reduced IFN- $\gamma$ levels and do not properly regulate NK cell activity [32]. These findings could contribute to the relationship between NK cell activity and immunological disorders by measuring IFN- $\gamma$ concentration in NK cells. The effects of CP and/or LABs on NK cell activity were confirmed using whole blood. Although there was no significant difference between the groups, both the L-200655 and D-200655 groups showed an increase in IFN- $\gamma$ concentration compared with the SC group. These results suggest that $L$. plantarum 200655 derived from kimchi can potentially recover NK cell activity.

The spleen is a crucial immune lymphoid organ in the body, with resident $\mathrm{T}$ and B lymphocytes as well as monocytes and macrophages for persistence of immunological homeostasis [33, 34]. LPS is a mitogen that causes cellular proliferation in B lymphocytes [35]. In this study, splenocytes from each group were co-incubated with LPS. This resulted in an increase in proinflammatory cytokines (TNF- $\alpha$ and IL-6) and mediators (iNOS and COX2 ) in both the L-200655 and D-200655 groups. Overall, the mRNA expression of TNF- $\alpha$, IL-6, IL-1 $\beta$, iNOS, and COX-2 was the highest in the L-200655 group. Although the MAPK signaling pathway was also activated in all groups compared with the SC group, L-200655 showed marked phosphorylation of both ERK and p38 MAPK. Notably, the SC group displayed increased phosphorylation of ERK, unlike p38 MAPK (Fig. 4). It could be considered that $\mathrm{CP}$ showed anti-tumor immunity through immune potentiation. A previous study described that the anti-tumor effects of CP depend on the dose administered and revealed immune potentiation or direct cytolytic activity [36]. Another study also reported that CP injection of $70 \mathrm{mg} / \mathrm{mouse}$ shows immune potentiation and expedites tumor growth in combination with an exosome-based vaccine against large established solid tumors [37]. Numerous intracellular signaling pathways are linked to inflammatory responses [38]. Among them, MAPK, including p38, ERK1/2, and JNK, regulate cell growth and development with cellular responses to cytokines and stresses [9], and MAPK pathways are accompanied by NF- $\mathrm{KB}$ for modulation of proinflammatory cytokines and inflammatory processes. In addition, many studies have reported that the regulation of both iNOS and COX-2 is associated with activation of the MAPK signaling pathway. In this study, the MAPK signaling pathway was an important molecular target of LAB derived from kimchi.

LAB exhibit multiple biological effects, including anti-obesity, anti-inflammatory, anti-staphylococcal, antiallergic, immune-enhancing, and antioxidant activities. Muhammad et al. described that, because probiotic effects are strain-specific, they do not behave via the same mechanisms [39]. The mechanism underlying the biological activities should be investigated in each relevant strain because the related response pathways vary. In addition, further study to examine why different effects appear in live and heat-killed LAB needs to be performed. As a potential reason, it could be considered that denaturation of the cell membrane composition is caused by heating, which results in various effects on immune responses. Chu-Chun Ou et al. reported that heat-treated $\mathrm{LAB}$ leads to longer expiration date, easier storage, and more stable transportation of product [40]. This could be seen as contributing to applicability in the food industry. Although many studies have shown that LAB originated from kimchi could attenuate immunocompromised status, to our knowledge, this is the first study to show that L. plantarum 200655-derived kimchi improves immunosuppression through regulation of the p38 MAPK/ERK signaling pathway in a CP-induced immunosuppression mouse model. However, a limitation of our study is that detailed mechanisms related to the p38 MAPK/ERK signaling pathway at the molecular level could not be demonstrated. Therefore, further studies are required to elucidate the exact mechanisms.

\section{Acknowledgments}

This work was supported by the Korea Institute of Planning and Evaluation for Technology in Food, Agriculture, and Forestry (IPET) through the Innovational Food Technology Development Program (\#1190093), funded by the Ministry of Agriculture, Food and Rural Affairs (MAFRA).

\section{Conflict of Interest}

The authors have no financial conflicts of interest to declare.

\section{References}

1. Shirani K, Hassani FV, Razavi-Azarkhiavi K, Heidari S, Zanjani BR, Karimi G. 2015. Phytotrapy of cyclophosphamide-induced immunosuppression. Environ. Toxicol. Pharmacol. 39: 1262-1275.

2. Do Youn Jeong, Hee Jong Yang, Su Ji Jeong, Min Guk Kim, Chi Young Yun, Hak Yong Lee, et al. 2019. Immunostimulatory effects of blueberry yeast fermented powder against cyclophosphamide-induced immunosuppressed model. Soc. Pathol. Korean Med. 33: 48-55. 
3. Lim TG, Jang M, Cho CW, Hong HD, Kim KT, Lee SY, et al. 2016. White ginseng extract induces immunomodulatory effects via the MKK4-JNK pathway. Food Sci. Biotechnol. 25: 1737-1744.

4. Vivier E, Raulet DH, Moretta A, Caligiuri MA, Zitvogel L, Lanier LL, et al. 2011. Innate or adaptive immunity? The example of natural killer cells. Science 331: 44-49.

5. Shen T, Wang G, You L, Zhang L, Ren H, Hu W, et al. 2017. Polysaccharide from wheat bran induces cytokine expression via the tolllike receptor 4-mediated p38 MAPK signaling pathway and prevents cyclophosphamide-induced immunosuppression in mice. Food Nutr. Res. 61: 1344523.

6. Ait-Oufella H, Sage AP, Mallat Z, Tedgui A. 2014. Adaptive (T and B cells) immunity and control by dendritic cells in atherosclerosis. Circ. Res. 114: 1640-1660.

7. Cheng C, Shou Q, Lang J, Jin L, Liu X, Tang D, et al. 2020. Gehua Jiecheng Decoction Inhibits Diethylnitrosamine-Induced Hepatocellular Carcinoma in Mice by Improving Tumor Immunosuppression Microenvironment. Front. Pharmacol. 11: 809.

8. Dong N, Li X, Xue C, Zhang L, Wang C, Xu X, et al. 2020. Astragalus polysaccharides alleviates LPS-induced inflammation via the NF-kappaB/MAPK signaling pathway. J. Cell Physiol. 235: 5525-5540.

9. Zhang W, Liu HT. 2002. MAPK signal pathways in the regulation of cell proliferation in mammalian cells. Cell Res. 12: 9-18.

10. Liu X, Ye F, Xiong H, Hu D, Limb GA, Xie T, et al. 2014. IL-1beta upregulates IL-8 production in human muller cells through activation of the p38 MAPK and ERK1/2 signaling pathways. Inflammation 37: 1486-1495.

11. Namba S, Nakano R, Kitanaka T, Kitanaka N, Nakayama T, Sugiya H. 2017. ERK2 and JNK1 contribute to TNF-alpha-induced IL-8 expression in synovial fibroblasts. PLoS One 12: e0182923.

12. Jung IS, Jeon MG, Oh DS, Jung YJ, Kim HS, Bae D, et al. 2019. Micronized, Heat-treated Lactobacillus plantarum LM1004 alleviates cyclophosphamide-induced immune suppression. J. Med. Food 22: 896-906.

13. Xu X, Zhang X. 2015. Effects of cyclophosphamide on immune system and gut microbiota in mice. Microbiol. Res. 171: 97-106.

14. Qi Q, Dong Z, Sun Y, Li S, Zhao Z. 2018. Protective effect of bergenin against cyclophosphamide-induced immunosuppression by immunomodulatory effect and antioxidation in Balb/c Mice. Molecules 23: 2668.

15. Salva S, Marranzino G, Villena J, Aguero G, Alvarez S. 2014. Probiotic Lactobacillus strains protect against myelosuppression and immunosuppression in cyclophosphamide-treated mice. Int. Immunopharmacol. 22: 209-221.

16. Jang SE, Joh EH, Lee HY, Ahn YT, Lee JH, Huh CS, et al. 2013. Lactobacillus plantarum HY7712 ameliorates cyclophosphamideinduced immunosuppression in mice. J. Microbiol. Biotechnol. 23: 414-421.

17. Hak-Jong Choi, Na-Kyoung Lee, Paik H-D. 2015. Health benefits of lactic acid bacteria isolated from kimchi, with respect to immunomodulatory effects. Food Sci. Biotechnol. 24: 783-789.

18. Ferreira Dos Santos T, Alves Melo T, Almeida ME, Passos Rezende R, Romano CC. 2016. Immunomodulatory effects of Lactobacillus plantarum Lp62 on intestinal epithelial and mononuclear cells. Biomed. Res. Int. 2016: 8404156.

19. Hessle C, Hanson LA, Wold AE. 1999. Lactobacilli from human gastrointestinal mucosa are strong stimulators of IL-12 production. Clin. Exp. Immunol. 116: 276-282.

20. Hoyong Lee, Young-Tae Ahn, Se-Hoon Park, Do-Young Park, Young-Woo Jin, Cha Soon Kim, et al. 2014. Lactobacillus plantarum HY7712 protects against the impairment of NK-cell activity caused by whole-body c-irradiation in mice. J. Microbiol. Biotechnol. 24: 127-131.

21. Jorjao AL, de Oliveira FE, Leao MV, Carvalho CA, Jorge AO, de Oliveira LD. 2015. Live and heat-killed Lactobacillus rhamnosus ATCC 7469 may induce modulatory cytokines profiles on macrophages RAW 264.7. Sci. WorldJ. 2015: 716749.

22. Stenman LK, Patterson E, Meunier J, Roman FJ, Lehtinen MJ. 2020. Strain specific stress-modulating effects of candidate probiotics: A systematic screening in a mouse model of chronic restraint stress. Behav. Brain Res. 379: 112376.

23. Yang SJ, Lee JE, Lim SM, Kim YJ, Lee NK, Paik HD. 2019. Antioxidant and immune-enhancing effects of probiotic Lactobacillus plantarum 200655 isolated from kimchi. Food Sci. Biotechnol. 28: 491-499.

24. Liu J, Sun H, Nie C, Ge W, Wang Y, Zhang W. 2018. Oligopeptide derived from solid-state fermented cottonseed meal significantly affect the immunomodulatory in BALB/c mice treated with cyclophosphamide. Food Sci. Biotechnol. 27: 1791-1799.

25. Cheng D, Wan Z, Zhang X, Li J, Li H, Wang C. 2017. Dietary Chlorella vulgaris ameliorates altered immunomodulatory functions in cyclophosphamide-induced immunosuppressive mice. Nutrients 9: 708.

26. Hyun-Ok Ku, Chang-Hee Kweon, Joon-Hyoung Cho, Sang-Hee Jeong, Shin-Ja Park, Yun-Bae Kim, et al. 1997. Thymocyte apoptosis induced by cyclophosphamide in rats. J. Toxicol. Public Health 13: 39-48.

27. Kang KS, Shin S, Lee SI. 2020. N-acetylcysteine modulates cyclophosphamide-induced immunosuppression, liver injury, and oxidative stress in miniature pigs. J. Anim. Sci. Technol. 62: 348-355.

28. Mansour DF, Saleh DO, Mostafa RE. 2017. Genistein ameliorates cyclophosphamide - induced hepatotoxicity by modulation of oxidative stress and inflammatory mediators. Open Access Maced. J. Med. Sci. 5: 836-843.

29. Burrack KS, Hart GT, Hamilton SE. 2019. Contributions of natural killer cells to the immune response against plasmodium. Malar. J. 18: 321 .

30. Lemire P, Galbas T, Thibodeau J, Segura M. 2017. Natural killer cell functions during the innate immune response to pathogenic Streptococci. Front. Microbiol. 8: 1196.

31. Wang R, Jaw JJ, Stutzman NC, Zou Z, Sun PD. 2012. Natural killer cell-produced IFN-gamma and TNF-alpha induce target cell cytolysis through up-regulation of ICAM-1. J. Leukoc. Biol. 91: 299-309.

32. Leonard Angka, Andre B Martel, Marisa Kilgour, Ahwon Jeong, Manahil Sadiq, Christiano Tanese de Souza, et al. 2018. Natural killer cell IFN $\gamma$ secretion is profoundly suppressed following colorectal cancer surgery. Ann. Surg. Oncol. 25: 3747-3754.

33. Lewis SM, Williams A, Eisenbarth SC. 2019. Structure and function of the immune system in the spleen. Sci. Immunol. 4: eaau6085.

34. Poon MML, Farber DL. 2020. The whole body as the system in systems immunology. iScience 23: 101509.

35. Xu H, Liew LN, Kuo IC, Huang CH, Goh DL, Chua KY. 2008. The modulatory effects of lipopolysaccharide-stimulated B cells on differential T-cell polarization. Immunology 125: 218-228.

36. Le DT, Jaffee EM. 2012. Regulatory T-cell modulation using cyclophosphamide in vaccine approaches: a current perspective. Cancer Res. 72: 3439-3444.

37. Brode S, Cooke A. 2008. Immune-potentiating effects of the chemotherapeutic drug cyclophosphamide. Crit. Rev. Immunol. 28: 109-126.

38. Lim HS, Kim YJ, Kim BY, Jeong SJ. 2019. Bakuchiol suppresses inflammatory responses via the downregulation of the p38 MAPK/ ERK signaling pathway. Int. J. Mol. Sci. 20: 3574 .

39. Amer M, Nadeem M, Nazir SUR, Fakhar M, Abid F, Ain QU, et al. 2018. Probiotics and their use in inflammatory bowel disease. Altern. Ther. Health Med. 24: 16-23.

40. Ou CC, Lin SL, Tsai JJ, Lin MY. 2011. Heat-killed lactic acid bacteria enhance immunomodulatory potential by skewing the immune response toward Th1 polarization. J. Food Sci. 76: M260-267. 